arising in shear layers also without the aid of these devices.

These experimental results are presented as evidence of the occurrence of peripherally periodic concentration of vorticity derived from the core of the parent vortex. Their occurrence could be related to radial divergence of the core region as postulated by linearized theory.

\section{ACKNOWLEDGMENTS}

The authors were favored to have the assistance of Vincent Neradka who contributed by his experimental skill, sustained effort, and grasp of objectives, to the accomplishment of the experimental program. The research was carried out under Grant from the Air Force Office of Scientific Research AFOSR $62-230$.

\title{
Velocity of a Fluid Mass Imbedded in Another Fluid Flowing in a Porous Medium
}

\author{
Chia-Shun YiH \\ The University of Michigan, Ann Arbor, Michigan,* and Huyck Felt Company
}

(Received 20 November 1962; revised manuscript received 16 May 1963)

\begin{abstract}
The velocity of a fluid mass imbedded in another fluid, which is of a different viscosity and a different specific weight and flowing in a porous medium under a prevailing uniform pressure gradient, is investigated. The fluid mass may take the form of a circular or elliptic cylinder, a sphere, or an ellipsoid, and the orientation of the fluid mass, if not spherical, is completely arbitrary with respect to both the direction of the pressure gradient and that of gravity. Exact closed solutions are obtained. The results for two-dimensional flows are applicable to Hele-Shaw cells.
\end{abstract}

\section{INTRODUCTION}

$\mathbf{I}^{\mathrm{N}}$ $\mathrm{N}$ this paper we deal with the velocity of a fluid mass imbedded in another fluid flowing in porous media. The fluid mass may take the form of a circular or elliptic cylinder, a sphere, or an ellipsoid. The viscosity and the specific weight of the fluid mass may differ from those of the ambient fluid, and the orientation of the cylinder, or of the ellipsoid, may be entirely arbitrary with respect to the direction of the prevailing pressure gradient and to the direction of gravity. Exact closed solutions for the velocity of the fluid mass are obtained for the cases of the elliptic cylinder and of the ellipsoid, and the solutions for a circular cylinder and for a sphere follow as special cases.

Polubarinova-Kochina and Falkovich ${ }^{1}$ referred to solutions for the velocity in porous media of a fluid mass in the form of an ellipsoid of revolution. These solutions were presented in a more general form in the work of Taylor and Saffman, ${ }^{2}$ who also considered the speed of a circular or elliptic bubble

\footnotetext{
* Permanent address.

${ }^{1}$ P. Y. Polubarinova-Kochina and S. B. Falkovich, Advances Appl. Mech. 2, 153, 1951.

${ }^{2}$ Sir Geoffrey Taylor and P. G. Saffman, Quart. J. Mech. and Appl. Math. 12, 2651959.
}

moving in a Hele-Shaw cell. Their solutions for these two cases are for two-dimensional flows only, because the velocity normal to the walls of the Hele-Shaw cell is necessarily zero. These solutions were obtained as limiting cases of a bubble moving in a Hele-Shaw cell of finite width, as the width approaches infinity, and not in the direct way used in this paper. The solution of Taylor and Saffman for the velocity of the elliptic bubble is further specialized in that the motion of the bubble is parallel to either one of its axes.

The main justification for writing this paper is that the solution for the general ellipsoid and the general solution for the elliptic cylinder are new, as far as the writer is aware, and that they bring out the very interesting behavior of ellipsoidal or elliptic-cylindrical masses of fluid in porous media. A minor justification is that the solutions for all the cases, general or special, are presented in more direct and explicit forms than hiterto. In principle, the solution for the elliptic cylinder can be derived from the one for the general ellipsoid. But in practice this derivation is not simple. Therefore the solution for the elliptic cylinder has been derived separately. The problems studied bear on the problem of ex- 


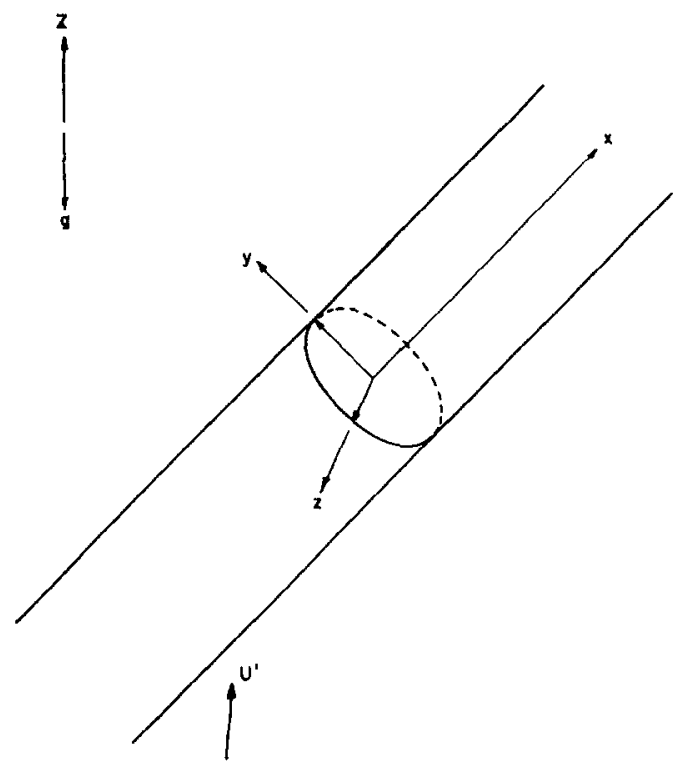

Frg. 1. Definition sketch for the case of an elliptic cylinder. The direction cosines of gravity is $\alpha, \beta$, and $\gamma$. Those of $U^{\prime}$ are $\alpha^{\prime}, \beta^{\prime}$, and $\gamma^{\prime}$.

traction of oil from the ground in the presence of water, and on the problem of water removal encountered in the paper industry. In the latter problem, air and water are exuded from a felt carrying a wet paper sheet and passing through two rollers, and it is important to find out how much faster the air moves relative to the water through the porous media of felt and paper sheet.

\section{GOVERNING DIFFERENTIAL SYSTEM}

Cartesian coordinates $x, y$, and $z$ will be used. The coordinate axes are fixed with respect to the fluid mass under consideration. The orientation of the axes will be specified in each case to be considered. The $x^{\prime}$ axis will be taken along the general direction of flow of the ambient fluid. The direction cosines of the $x^{\prime}$ axis with respect to the $x, y$, and $z$ coordinates will be denoted by $\alpha^{\prime}, \beta^{\prime}$, and $\gamma^{\prime}$. The $z$ axis will be taken in a direction opposite to that of the gravitational acceleration. Its direction cosines will be denoted by $\alpha, \beta$, and $\gamma$.

Seepage flow of a fluid in porous media is governed by Darcy's law, which states that

$$
\frac{\mu}{k}(u, v, w)=-\left(\frac{\partial}{\partial x} \frac{\partial}{\partial y} \frac{\partial}{\partial z}\right)(p+\rho g Z),
$$

in which $\mu$ is the viscosity of the fluid, $\rho$ is its density, $k$ is the permeability of the porous medium, assumed constant, $p$ is the pressure, $g$ is the gravitational acceleration, and $u, v$, and $w$ are the velocity components in the directions of increasing $x, y$, and $z$, respectively. The equation of continuity is, if the fluid is incompressible,

$$
\frac{\partial u}{\partial x}+\frac{\partial v}{\partial y}+\frac{\partial w}{\partial z}=0 .
$$

If $\mu$ and $k$ are constant, (1) and (2) can be combined to form the single equation

$$
\left(\frac{\partial^{2}}{\partial x^{2}}+\frac{\partial^{2}}{\partial y^{2}}+\frac{\partial^{2}}{\partial z^{2}}\right) \phi=0,
$$

in which

$$
\phi=p+\rho g Z
$$

is a potential.

One remark needs to be made on the coordinates used. Since the porous medium is at rest, application of Darcy's law requires that the coordinates be fixed, otherwise (1) would give, for example, no pressure drop in uniform flow with a coordinate system moving with it. On the other hand, for the unsteady flows caused by moving bodies, such as are treated here, it is not convenient to use a coordinate system which is fixed once and for all. The dilemma is resolved by the use of fixed coordinates coinciding instantaneously with the coordinates most natural and convenient for the body. Since (3), the boundary conditions (as will be seen), and hence the solution are entirely independent of the history of the motion, the use of instantaneous coordinates is justified. However, these are, in concept, fixed coordinates.

The most important fact about (3) is that it is linear. One of the conditions at the boundary of two fluids in contact is that $p$ should be continuous. From (4) it can be seen that this condition will be linear in $\phi$, although not necessarily homogeneous in $\phi$. The linearity of (3) and the boundary conditions allow solutions to be superposed, provided any nonhomogeneity of the boundary conditions in $\phi$ is properly taken into account. Since the interface of the two fluids is not specified a priori, it is necessary to have another boundary condition at the interface. That condition is a kinematic one, and follows from the fact that the velocity component normal to the interface must be the same for both fluids. Since the flow is governed by the Laplace equation, the tangential velocities at the interface will not be the same for the two fluids. In reality, the tangential velocity changes from one value for one fluid to another for the other fluid in a very short distance comparable to the pore size of the medium. Hence the slippage at the interface is an idealized situation closely representing reality. 


\section{VELOCITY OF A CYLINDRICAL MASS OF ELLIPTIC CROSS SECTION}

The $x$ axis will be assumed to coincide with the axis of the elliptic cylinder. The $y$ axis coincides with the major axis, and the $z$ axis with the minor axis of the ellipse

$$
y^{2} / a^{2}+z^{2} / b^{2}=1,
$$

which describes the cross section of the elliptic cylinder under consideration.

Consider now a flow of the ambient fluid with velocity $U^{\prime}$ in the $x^{\prime}$ direction at infinity. The component of $U^{\prime}$ in the $x$ direction is $U_{1}=\alpha^{\prime} U^{\prime}$. Since

$$
\partial Z / \partial x=\alpha,
$$

the first of equations (1) can be written

$$
(\mu / k) u=-\partial p / \partial x-\rho g \alpha .
$$

For the ambient fluid,

$$
-\partial p / \partial x=\left(\mu_{1} / k\right) U_{1}+\rho_{1} g \alpha .
$$

For the fluid mass,

$$
-\partial p / \partial x=\left(\mu_{2} / k\right) U_{2}+\rho_{2} g \alpha .
$$

Hence the continuity of $p$ at the interface demands that

$$
U_{2}=\left(\mu_{1} / \mu_{2}\right) U_{1}+k\left(\rho_{1}-\rho_{2}\right) g \alpha / \mu_{2} .
$$

This is in fact true whatever the cross section of the cylinder. For $\left(\rho_{1}-\rho_{2}\right) \alpha=0$, (6) reduces to

$$
U_{2}=\left(\mu_{1} / \mu_{2}\right) U_{1} \text {, }
$$

in agreement with the previous result of $\mathrm{Yih}^{3}{ }^{3}$

For the motion of the cylinder in the directions of $y$ and $z$, it is advantageous to introduce the elliptic coordinates $\xi$ and $\eta$. They are connected with $y$ and $z$ by

or

$$
y+i z=c \cosh (\xi+i \eta),
$$

$$
y=c \cosh \xi \cos \eta, \quad z=c \sinh \xi \sin \eta .
$$

On the ellipse, $\xi=\xi_{0}$, because (5) and (8) coincide with

$$
a=c \cosh \xi_{0}, \quad b=c \sinh \xi_{0},
$$

which defines $c$ and $\xi_{0}$ in terms of $a$ and $b$. The flow caused by a velocity $\Delta V$ in the $y$ direction relative to the ambient fluid can be described by

$$
\phi+i \psi=C e^{-(\xi+i n)},
$$

in which $\psi$ is the stream function, and is conjugate to $\phi$. If, as it will turn out to be the case, the fluid cylinder moves as a solid body, the kinematic boundary condition on the ellipse is

$$
\psi=-\Delta V z+\text { constant, }
$$

\footnotetext{
C.-S. Yih, J. Fluid Mech. 10, 133, 1961
}

if $\Delta U, \Delta V$, and $\Delta W$ are the components of the velocity of the fluid mass relative to the ambient fluid. This condition follows from the requirement that the velocity component normal to the surface of the fluid mass must be the same for the mass and for the ambient fluid. Since

$$
\psi=-C e^{-\xi} \sin \eta,
$$

this boundary condition is satisfied if

$$
C e^{-\xi_{0}}=\Delta V c \sinh \xi_{0} .
$$

Thus

$$
C=\frac{\Delta V b c}{a-b}=\Delta V b\left(\frac{a+b}{a-b}\right)^{\frac{1}{2}}
$$

and

On the ellipse

$$
\phi=C e^{-\xi_{0}} \cos \eta=\Delta V\left(\tanh \xi_{0}\right) y=\frac{b}{a} \Delta V y .
$$

Similarly, if the velocity in the $z$ direction of the cylinder relative to the ambient fluid is $\Delta W$,

$$
\phi+i \psi=i D e^{-(\xi+i \eta)},
$$

with

$$
D=\Delta W a[(a+b) /(a-b)]^{\frac{1}{2}},
$$

and

$$
\phi=(a / b) \Delta W z .
$$

The components of the velocity $U^{\prime}$ in the coordinate directions are $\alpha^{\prime} U^{\prime}, \beta^{\prime} U^{\prime}$, and $\gamma^{\prime} U^{\prime}$. These will be denoted by $U_{1}, V_{1}$, and $W_{1}$. Now, on the ellipse, with $\Delta V=V_{2}-V_{1}$, restoring the factor $\mu / k$,

$$
\begin{aligned}
& \phi_{1}=\left(\mu_{1} / k\right)\left[-V_{1} y+\left(V_{2}-V_{1}\right) y b / a\right], \\
& \phi_{2}=-\left(\mu_{2} / k\right) V_{2} y,
\end{aligned}
$$

if the elliptic cylinder is assumed to move as a solid body with velocity components $U_{2}, V_{2}$, and $W_{2}$. Continuity of $p$ then demands that

$\left(\mu_{1} / k\right)\left[-V_{1} y+\left(V_{2}-V_{1}\right) y b / a\right]-\rho_{1} g \beta y$

which gives

$$
=-\left(\mu_{2} / k\right) V_{2} y-\rho_{2} g \beta y \text {, }
$$

$$
V_{2}=\frac{\mu_{1}(a+b)}{\mu_{1} b+\mu_{2} a} V_{1}+\frac{a k\left(\rho_{1}-\rho_{2}\right) g \beta}{\mu_{1} b+\mu_{2} a} .
$$

Similarly,

$$
W_{2}=\frac{\mu_{1}(a+b)}{\mu_{1} a+\mu_{2} b} W_{1}+\frac{b k\left(\rho_{1}-\rho_{2}\right) g \gamma}{\mu_{1} a+\mu_{2} b} .
$$

The expression for $U_{2}$ is still given by (6). From (6), (14), and (15) it can be seen that the velocity of the cylinder is independent of its size, although it does depend on its shape.

If $\rho_{1}=\rho_{2}$, then 


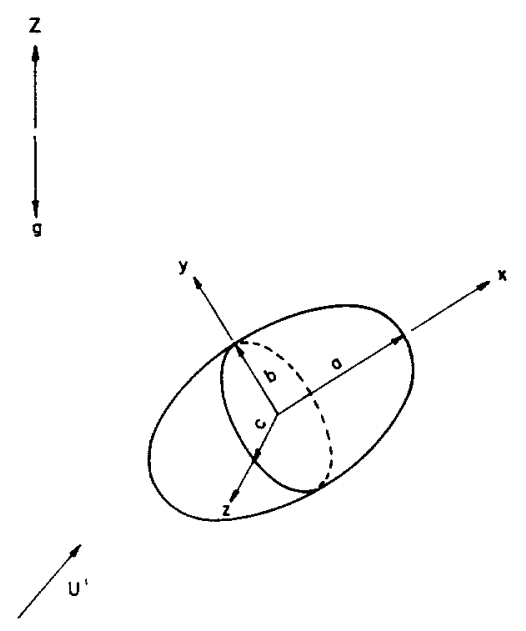

Fig. 2. Definition sketch for the case of an ellipsoid. The direction cosines of gravity and of $U^{\prime}$ remain the same as in Fig. 1.

$$
\begin{aligned}
\frac{W_{2}}{V_{2}} & =\frac{\mu_{1} b+\mu_{2} a}{\mu_{1} a+\mu_{2} b} \frac{W_{1}}{V_{1}} \\
& =\left[1+\frac{(a-b)\left(\mu_{2}-\mu_{1}\right)}{\mu_{1} a+\mu_{2} b}\right] \frac{W_{1}}{V_{1}} .
\end{aligned}
$$

If $\mu_{2}<\mu_{1}$, then since $a>b$, we have

$$
W_{2} / V_{2}<W_{1} / V_{1}
$$

so that in the $y z$ plane the cylinder will not move in the same direction with the ambient fluid, but will move more closely to the major axis of the ellipse. The reverse is true if $\mu_{2}>\mu_{1}$. This is indeed a very interesting situation.

If $U^{\prime}=0$, then

$$
U_{2}: V_{2}: W_{2}=\left(\mu_{1} b+\mu_{2} a\right) \alpha: \mu_{2} a \beta: \mu_{2} b \gamma,
$$

so that the cylinder will not move in the direction of gravity, but will have a velocity deviating from that direction toward the $y$ axis, and even more toward the $x$ axis. In other words, it will drift in such a way as to favor the axes of the cylinder in the order of their length: $\infty, a$, and $b$.

The solution for the special case of a circular cylinder can be derived from (13) and (14). The results are $(6)$,

$$
V_{2}=\frac{2 \mu_{1}}{\mu_{1}+\mu_{2}} V_{1}+\frac{k\left(\rho_{1}-\rho_{2}\right)}{\mu_{1}+\mu_{2}} g \beta
$$

and

$$
W_{2}=\frac{2 \mu_{1}}{\mu_{1}+\mu_{2}} W_{1}+\frac{l\left(\rho_{1}-\rho_{2}\right) g \gamma}{\mu_{1}+\mu_{2}} .
$$

For $\mu_{2}=0$ and $(\rho-\rho) \beta=0, V=2 V$, in agreement with the result of Taylor and Saffman. ${ }^{2}$

From (6), (16), and (17), it can be seen that the velocity of the cylinder is entirely independent of its size, and that, when the ambient fluid is at rest (i.e., if $U^{\prime}=0$ ), the velocity of the cylinder is simply $k\left(\rho_{1}-\rho_{2}\right) g /\left(\mu_{1}+\mu_{2}\right)$, in the direction opposite to that of gravity if $\rho_{1}>\rho_{2}$, and $k\left(\rho_{2}-\rho_{1}\right) g /\left(\mu_{1}+\mu_{2}\right)$ in the direction of gravity if $\rho_{2}>\rho_{1}$.

\section{THE VELOCITY OF AN ELLIPSOIDAL MASS}

Let the coordinate axes be taken along the axes of the ellipsoid, and the origin be located at its center. Then the fluid mass is an ellipsoid the surface of which is described by

$$
x^{2} / a^{2}+y^{2} / b^{2}+z^{2} / c^{2}=1,
$$

and the potentials for a velocity $\Delta U, \Delta V$, and $\Delta W$ of the ellipsoidal mass relative to the ambient fluid is, respectively,

$$
\begin{aligned}
& \phi=\frac{a b c}{2-\alpha_{0}} \Delta U x \int_{\lambda}^{\infty} \frac{d \lambda}{\left(a^{2}+\lambda\right) \Delta}, \\
& \phi=\frac{a b c}{2-\beta_{0}} \Delta V y \int_{\lambda}^{\infty} \frac{d \lambda}{\left(b^{2}+\lambda\right) \Delta},
\end{aligned}
$$

and

$$
\phi=\frac{a b c}{2-\gamma_{0}} \Delta W z \int_{\lambda}^{\infty} \frac{d \lambda}{\left(c^{2}+\lambda\right) \Delta},
$$

in which

$\alpha_{0}=a b c \int_{0}^{\infty} \frac{d \lambda}{\left(a^{2}+\lambda\right) \Delta}, \quad \beta_{0}=a b c \int_{0}^{\infty} \frac{d \lambda}{\left(b^{2}+\lambda\right) \Delta}$, $\gamma_{0}=a b c \int_{0}^{\infty} \frac{d \lambda}{\left(c^{2}+\lambda\right) \mathbf{\Delta}}$

and

$$
\Delta=\left[\left(a^{2}+\lambda\right)\left(b^{2}+\lambda\right)\left(c^{2}+\lambda\right)\right]^{\frac{1}{2}} .
$$

The coordinate $\lambda$ is the first of the so-called ellipsoidal coordinates $\lambda, \mu$, and $\nu$, which are roots of the cubic equation in $\theta$,

$$
\frac{x^{2}}{a^{2}+\theta}+\frac{y^{2}}{b^{2}+\theta}+\frac{z^{2}}{c^{2}+\theta}=1,
$$

for every point $(x, y, z)$. For the ellipsoid given by (18), $\lambda=0$ on the surface. On the assumption that the ellipsoid moves as a solid body, the kinematical boundary condition at the interface (that the normal velocity must be the same) is satisfied by the solutions (19), (20), and (21). On the ellipsoid,

$\phi=\frac{\alpha_{0}}{2-\alpha_{0}} \Delta U x, \phi=\frac{\beta_{0}}{2-\beta_{0}} \Delta V y, \phi=\frac{\gamma_{0}}{2-\gamma_{0}} \Delta W z$,

for the three modes of motion. For the motion parallel to the $x$ axis,

$$
\phi=\frac{\mu_{1}}{k}\left[-U_{1} x+\frac{\alpha_{0}}{2-\alpha_{0}}\left(U_{2}-U_{1}\right) x\right]
$$

for the ambient fluid and

$$
\phi=-\left(\mu_{2} / k\right) U_{2} x
$$

for the ellipsoidal fluid mass, assumed in solid body translation. Hence the continuity of pressure at the 
surface of the ellipsoid demands that

$$
\begin{array}{r}
\frac{\mu_{1}}{k}\left[-U_{1} x+\frac{\alpha_{0}}{2-\alpha_{0}}\left(U_{2}-U_{1}\right) x\right]-g \rho_{1} \alpha x \\
=-\left(\mu_{2} / k\right) U_{2} x-g \rho_{2} \alpha x,
\end{array}
$$

so that

$$
U_{2}=\frac{2 \mu_{1} U_{1}+\left(2-\alpha_{0}\right) k g\left(\rho_{1}-\rho_{2}\right) \alpha}{\alpha_{0} \mu_{1}+\left(2-\alpha_{0}\right) \mu_{2}} .
$$

Similarly,

$$
V_{2}=\frac{2 \mu_{1} V_{1}+\left(2-\beta_{0}\right) k g\left(\rho_{1}-\rho_{2}\right) \beta}{\beta_{0} \mu_{1}+\left(2-\beta_{0}\right) \mu_{2}},
$$

and

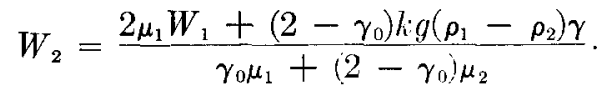

For the case $\rho_{1}=\rho_{2}$,

$$
U_{2} / U_{1}>V_{2} / V_{1}>W_{2} / W_{1}
$$

if $\mu_{2}<\mu_{1}$, and

$$
U_{2} / U_{1}<V_{2} / V_{1}<W_{2} / W_{1}
$$

if $\mu_{2}>\mu_{1}$, since $\alpha_{0}<\beta_{0}<\gamma_{0}$. This is indeed a very amusing situation. For the case $U^{\prime}=0$, so that $U_{1}=V_{1}=W_{1}=0$,

$$
U_{2}: V_{2}: W_{2}=\frac{\alpha}{\left[\alpha_{0} /\left(2-\alpha_{0}\right)\right] \mu_{1}+\mu_{2}}: \frac{\beta}{\left[\beta_{0} /\left(2-\beta_{0}\right)\right] \mu_{1}+\mu_{2}}: \frac{\gamma}{\left[\gamma_{0} /\left(2-\gamma_{0}\right)\right] \mu_{1}+\mu_{2}} .
$$

Since $\alpha_{0}<\beta_{0}<\gamma_{0}$, the direction of the velocity of the ellipsoid will deviate from that of gravity toward the directions of the longer axes, i.e., toward the direction of $y$ and even more toward the direction of $x$, whether $\mu_{2}$ is greater or less than $\mu_{1}$. The independence of the sign of $\mu_{1}-\mu_{2}$ is due to the fact that the inequality of the three axes is not merely a measure of the inequality of the resistance to flow, as in the case $\rho_{1}=\rho_{2}$, but is also a measure of the inequality of the motive force in the three directions, when $\rho_{1} \neq \rho_{2}$.

For the special case of a sphere, $\alpha_{0}=\beta_{0}=\gamma_{0}=\frac{2}{3}$. Hence (24)-(26) become

$$
\begin{aligned}
\left(U_{2}, V_{2}, W_{2}\right)= & \frac{3 \mu_{1}}{2 \mu_{2}+\mu_{1}}\left(U_{1}, V_{1}, W_{1}\right) \\
& \quad+\frac{2 k g\left(\rho_{1}-\rho_{2}\right)}{2 \mu_{2}+\mu_{1}}(\alpha, \beta, \gamma) .
\end{aligned}
$$

If $\mu_{2}=0$ and $\rho_{1}=\rho_{2}, U_{2}=3 U_{1}$, in agreement with Taylor and Saffman's result. If $U^{\prime}=0$, the sphere moves in the direction of gravity or in a direction opposite to it, according as $\rho_{2}>\rho_{1}$ or $\rho_{2}<\rho_{1}$. In either case the speed is

$$
2 k g\left|\rho_{1}-\rho_{2}\right| /\left(2 \mu_{2}+\mu_{1}\right) \text {. }
$$

\section{DISCUSSION}

In Secs. 3 and 4, the fluid mass has been assumed to move as a solid body, hence without change of form, and a solution is possible because, in the case of an elliptic cylinder, $y$ can be cancelled in (13), and, in the case of an ellipsoid, $x$ can be cancelled in (23). These are very special situations, and the solutions obtained are very probably the only ones corresponding to solid-body motion of the fluid mass. For a mass of given volume (or given volume per unit length), the solution is obviously not unique, for the mass can take infinitely many shapes. The stability of the fluid motion corresponding to these solutions is another question. It is known that large flat masses moving broadside on are not stable, but circular masses in Hele-Shaw cells have been observed to preserve their form and to move as a solid body. Perhaps surface tension will make the masses describe here stable, provided they are not too large in any dimension.

\section{APPLICATION TO HELE-SHAW CELLS}

Since Hele-Shaw cells provide a means of experimentation on flows in porous media, it may be mentioned that the results of Sec. 3 can be verified in the laboratory by the use of a Hele-Shaw cell. The flow is necessarily two dimensional, since the axis of the cylinder is necessarily perpendicular to its direction of motion. The direction of gravity may be related in any arbitrary fashion to the direction of the general flow of the ambient fluid and to the direction of the axis of the cylinder. However, gravity effects in the direction of the cylinder must be ignored, since the walls of the cell prohibit any flow in that direction. The error is a small since the spacing of the walls of the cell is supposed to be so small that any change of hydrostatic pressure over the thickness is small compared with the change of pressure from place to place in the cell, in a plane parallel to the walls.

\section{ACKNOWLEDGMENTS}

The author wishes to thank Dr. D. Wicker, Mr. J. Hintermaier, and Mr. S. J. McNamara, all of Huyck Felt Company, for their interest in this problem, and for the stimulation of Mr. McNamara's experiments. 